\title{
Human Adenovirus Associated with Severe Respiratory Infection, Oregon, USA, 2013-2014
}

\author{
Magdalena Kendall Scott, Christina Chommanard, Xiaoyan Lu, Dianna Appelgate, LaDonna Grenz, \\ Eileen Schneider, Susan I. Gerber, Dean D. Erdman, Ann Thomas
}

\begin{abstract}
Several human adenoviruses (HAdVs) can cause respiratory infections, some severe. HAdV-B7, which can cause severe respiratory disease, has not been recently reported in the United States but is reemerging in Asia. During October 2013-July 2014, Oregon health authorities identified 198 persons with respiratory symptoms and an HAdV-positive respiratory tract specimen. Among 136 (69\%) hospitalized persons, $31 \%$ were admitted to the intensive care unit and $18 \%$ required mechanical ventilation; 5 patients died. Molecular typing of 109 specimens showed that most (59\%) were HAdV-B7, followed by HAdVs-C1, -C2, -C5 (26\%); HAdVsB3, -B21 (15\%); and HAdV-E4 (1\%). Molecular analysis of 7 HAdV-B7 isolates identified the virus as genome type d, a strain previously identified only among strains circulating in Asia. Patients with HAdV-B7 were significantly more likely than those without HAdV-B7 to be adults and to have longer hospital stays. HAdV-B7 might be reemerging in the United States, and clinicians should consider HAdV in persons with severe respiratory infection.
\end{abstract}

$\mathrm{H}$ uman adenoviruses (HAdVs) are a common cause of respiratory infection in persons of all ages. Acute upper and lower respiratory tract diseases, including pneumonia and bronchitis, have been attributed to HAdVs. Although many infections are mild, some persons, such as very young children, elderly or immunocompromised persons, or persons who have underlying pulmonary or cardiac disease, might be at increased risk for severe disease (1-4). HAdV infection can occur sporadically, endemically, or epidemically and often is influenced by HAdV species and type (4). Common settings for infection include the community, military recruit training centers, hospitals, and chronic care facilities $(3,5-8)$.

Author affiliations: Oregon Public Health Division, Portland, Oregon, USA (M. Kendall Scott, A. Thomas); Centers for Disease Control and Prevention, Atlanta, Georgia, USA (C. Chommanard, X. Lu, E. Schneider, S.I. Gerber, D.D. Erdman); PeaceHealth Department of Quality and Improvement, Springfield, Oregon, USA (D. Appelgate); Oregon Public Health Laboratory, Hillsboro, Oregon, USA (L. Grenz)

DOI: http://dx.doi.org/10.3201/eid2206.151898
HAdV comprises 7 species (A-G), and $>51$ types have been characterized by immunotypic and molecular methods. HAdVs that are most often associated with symptomatic respiratory infections include species B (types 3, 7, 14, and 21), species C (types 1,2, and 5), and species E (type 4) (9). Infections with HAdV-C often are endemic, mild, and most commonly seen in young children (2). HAdVs can be shed from the respiratory and gastrointestinal tracts for weeks or longer, even in persons who are no longer symptomatic, especially young children and immunocompromised persons (10). In comparison, HAdV-B- and HAdV-E-associated respiratory infections are more commonly seen as part of an epidemic or as sporadic cases in adults, and infections with these viruses are often more severe (11).

Circulating HAdVs can vary temporally and geographically; emergent genomic variants are possibly associated with more severe illness $(8,12,13)$. Recent reports have noted severe respiratory disease associated with the reemergence of $\mathrm{HAdV}-\mathrm{B} 7$ and genomic variant $7 \mathrm{~d}$ in China and other countries in Asia (14-16). However, HAdVB7 was rarely reported in the United States during the past decade $(17,18)$. Among a convenience sample of 291 specimens sent to the Centers for Disease Control and Prevention (CDC) from mid-2004 through 2013 for HAdV typing, only $7(2.4 \%)$ were identified as HAdV-B7 (D.D. Erdman, pers. comm.).

In March 2014, clinicians in the metropolitan areas of Eugene and Portland, Oregon, USA, reported an increase in the number of HAdV detections among specimens from hospitalized patients with severe respiratory infections to the Oregon Public Health Division (OPHD). In this study, we describe the clinical, epidemiologic, and viral molecular features of this cluster of HAdV-positive cases identified during October 2013-July 2014.

\section{Methods}

OPHD asked 3 major hospital systems that perform HAdV diagnostic testing to participate in the investigation. The hospital systems comprised 14 facilities located primarily within the Eugene and Portland metropolitan areas. 
Facilities averaged 210 beds (range 25-688) and 11,774 admissions annually (range 1,263-35,614). These hospital systems were asked to provide information on HAdV-positive specimens from patients with respiratory infections diagnosed after September 2013. Requested information comprised basic demographic and clinical data and whether specimens were available for HAdV typing. On April 19, 2014, OPHD released a statewide notice through the Health Alert Network requesting providers in Oregon to consider HAdV in the differential diagnosis for patients presenting with severe pneumonia or for unusual clusters of pneumonia.

We also obtained historical HAdV detection data from Oregon clinical laboratories that reported to the National Respiratory and Enteric Virus Surveillance System to review recent trends in HAdV detections. In addition, 2 large Oregon hospital systems with available HAdV detection data compared HAdV detections for November 2013-April 2014 with those for November-April from the respiratory disease seasons of the previous 3 years (i.e., 2010-11, 2011-12, 2012-13).

The study population comprised persons in whom HAdV was detected in respiratory specimens during October 2013-July 2014 by hospital laboratories that perform virus isolation, direct fluorescent antibody, or PCR for HAdV detection (retrospective and prospective) and were willing to submit data to OPHD and CDC. A case-patient was defined as a person with respiratory symptoms and a positive HAdV laboratory test result. Illness was defined as severe if the patient was hospitalized.

We reviewed available medical records and collected the following information: basic demographic information, symptom onset date, symptoms, hospital admission and discharge dates, intensive care unit (ICU) admission, use of mechanical ventilation, specimen type and collection date, and HAdV laboratory results. The medical record review was conducted under Oregon's special study statute for issues of public health significance (http://arcweb.sos.state.or.us/pages/rules/oars_300/ oar_333/333 019.html).

Available clinical specimens were shipped to the Oregon State Public Health Laboratory and then submitted to the CDC for HAdV confirmation and molecular typing. HAdV-positive specimens were typed by either conventional PCR and sequencing of HAdV hexon gene hypervariable regions $1-6$ or by HAdV type-specific real-time PCR assays, as previously described (19,20). For enhanced genetic comparisons, genomic sequencing was performed on 7 HAdV-B7-positive samples collected during January 2014-May 2014. Deep sequencing libraries were prepared by using the Nextera XT DNA Sample Prep Kit and sequenced (250-bp paired-end sequencing) on an Illumina MiSeq Desktop Sequencer (both from Illumina, San Diego,
CA, USA) (protocol available on request). In silico genome restriction enzyme digestion profiles were generated by using NEBcutter V2.0 (21), and genome-type determinations were based on the classification system previously described (22). Sequences were compared with HAdV-B7 genome type d (HAdV-B7d) reference strains 0901HZ/ Shx/CHN/2009 (GenBank accession no. JF800905.1) and human/CHN/DG01/2011/7[P7H7F7] (GenBank accession no. KC440171.1). We used sequence alignment and neighbor-joining phylogenetic tree construction to compare the phylogenetic relationships among a representative sample of HAdV-B7 genomic sequences using ClustalW implemented in BioEdit version 7.0.5 (23) and MEGA7, respectively (24). We excluded cases determined by CDC to be HAdV-negative.

We used Excel (Microsoft Corp., Redmond, WA, USA) for data entry and SAS version 9.3 (SAS Institute, Cary, NC, USA) for data analysis. Mantel-Haenszel $\chi^{2}$ was used to assess associations. A p value $<0.05$ was considered significant.

\section{Results}

Comparison to historical data for the past 3 years demonstrated an increase in HAdV reports for November 2013April 2014. We found 2-fold and 9-fold increases in HAdV detections for the 2 large Oregon hospital systems for which historical data were available (Figure 1). HAdV detections from laboratories in Oregon reporting to the National Respiratory and Enteric Virus Surveillance System (Figure 2) increased 11-fold during the same period.

For October 9, 2013-July 7, 2014, we identified 198 patients who had an HAdV-positive respiratory specimen. Most were reported from hospital systems in Portland and surrounding counties (56\%), followed by Eugene and surrounding counties (39\%), Medford (4\%), and 2 other locations (1\%). Most (97\%) cases came from 3 major hospital systems in Oregon (hospital system A, 77 [39\%] cases; hospital system B, 69 [35\%]; hospital system C, 46 [23\%]); the remaining 6 cases came from a variety of sources around the state. Most (91\%) cases occurred in residents of Oregon; the remainder occurred in residents of Washington (7\%), and California (2\%). Case-patients ranged in age from 3 weeks to 80 years (median 8 years); $60 \%$ were male. For most $(87 \%)$ HAdV detections, symptom onset occurred or specimens were collected during JanuaryApril 2014 (Figure 3).

Specimens from $109(55 \%)$ HAdV patients were available for HAdV typing (Table 1). We identified 7 HAdV types; HAdV-B7 was most commonly detected (64 [59\%] specimens), followed by HAdV-C2 (14 [13\%]), HAdV-B21 (10 [9\%]), HAdV-C1 (10 [9\%]), HAdV-B3 (6 [6\%]), HAdV-C5 (2 [2\%]), and HAdV-E4 (1 [1\%]). In $2(2 \%)$ cases, HAdV-C1 and -C2 were co-detected. 
A

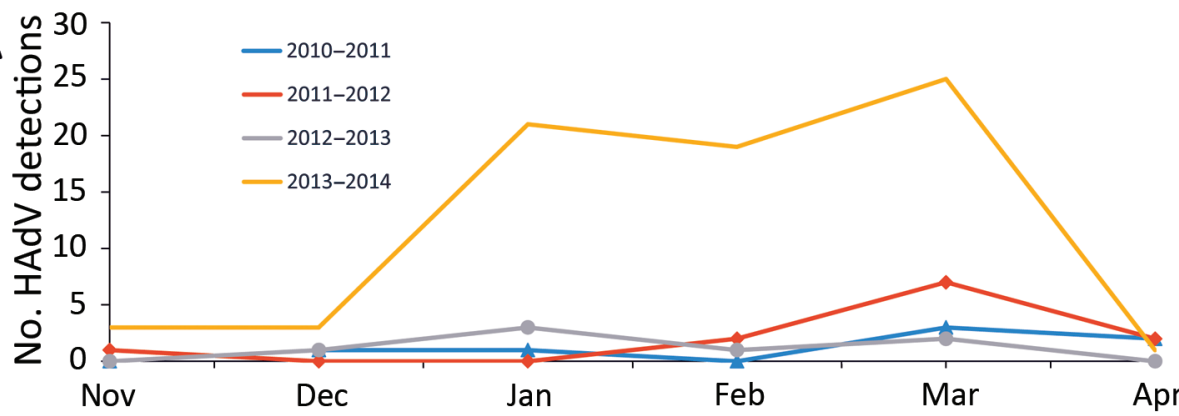

B

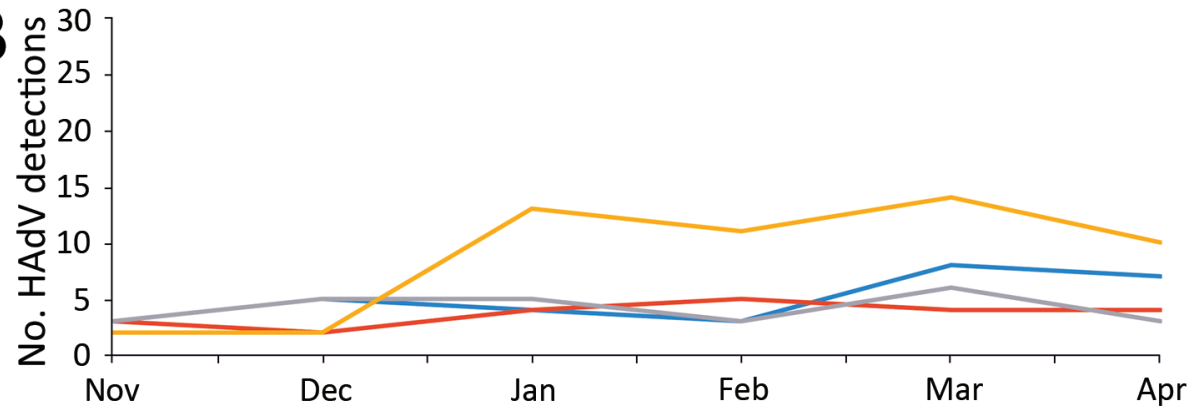

Figure 1. HAdV detections from 2 major hospital systems (A and B), Oregon, USA, NovemberApril 2010-2014. Historical data collected by the Oregon Public Health Division. Data for hospital system $C$ were not available. HAdV, human adenovirus.
The genome from 7 HAdV-B7-positive samples collected during January-May 2014 (GenBank accession no. KT963081) showed $>99.9 \%$ sequence identity and gave identical in silico $B a m H I$ and $B c l I$ restriction profiles with a HAdV-B7d variant circulating in China in 2009 and 2011 (22) (Figure 4). Specimens from 89 (45\%) HAdVpositive patients were not available for typing; $80 \%$ of these specimens came from hospital system A. HAdV type results were grouped by species (i.e., HAdV-C and HAdV-E), except for species B, which was grouped by type (i.e., HAdV-B7 and HAdVs-B3, -B21) to better highlight specific features of the rarely reported HAdV-B7. Most (74\%) specimens tested were upper respiratory tract specimens (i.e., nasopharyngeal wash or nasopharyngeal swab samples). Case-patients with HAdV-C1, -C2, or -C5 detections were generally younger (median age 1.2 years) than those with HAdVs-B3 or -B21 (median age 24.0 years) and HAdV-B7 (median age 20.0 years) (Table 1). Case-patients with HAdV-B7 were significantly older than those with non-HAdV-B7 (Table 2).

For all case-patients, the most common symptoms were fever (75\%), cough (61\%), shortness of breath (26\%), nausea or vomiting (24\%), and rhinorrhea (22\%) (Table 1). Pneumonia was reported for $32 \%$ of the $198 \mathrm{HAdV}$ patients ( $41 \%$ of those with HAdV-B $7,31 \%$ of those with HAdVsB3 or $-\mathrm{B} 21,21 \%$ of those with HAdVs-C1, $-\mathrm{C} 2$, or $-\mathrm{C} 5)$. A

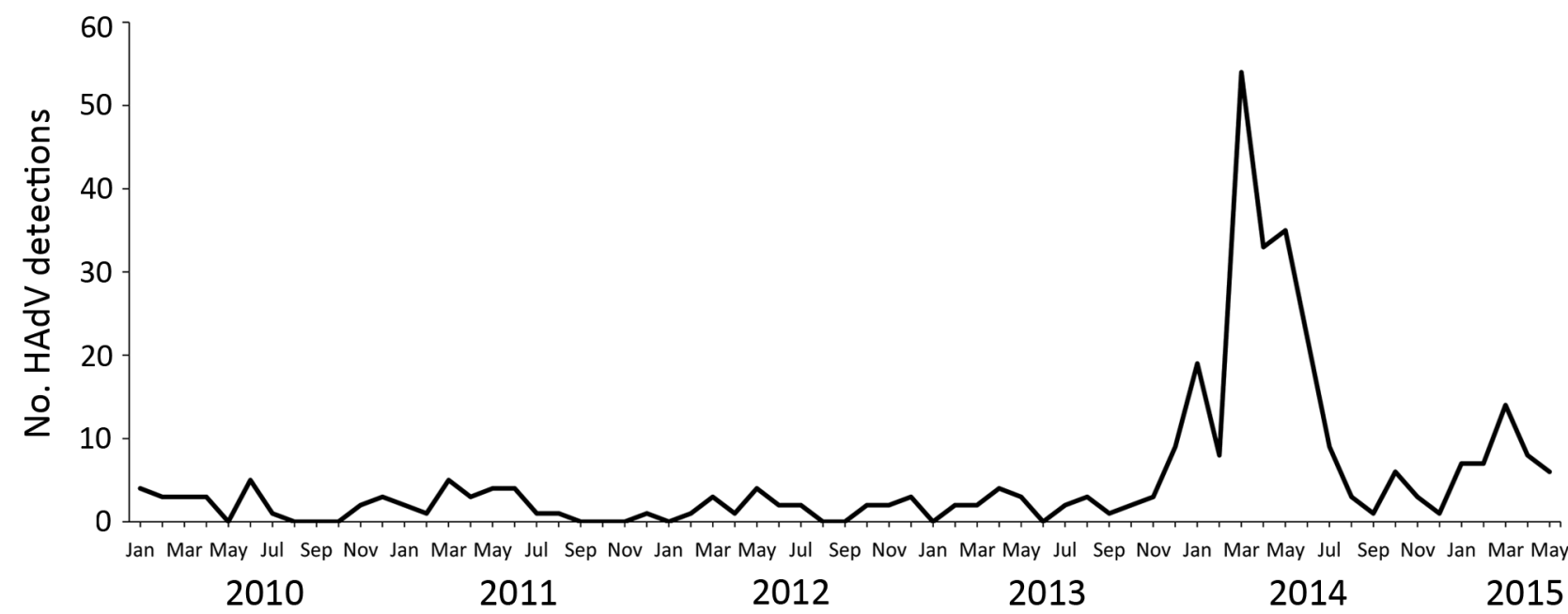

Figure 2. HAdV detections reported to the National Respiratory and Enteric Virus Surveillance System, Oregon, January $2010-M a y 2015$. HAdV, human adenovirus. 


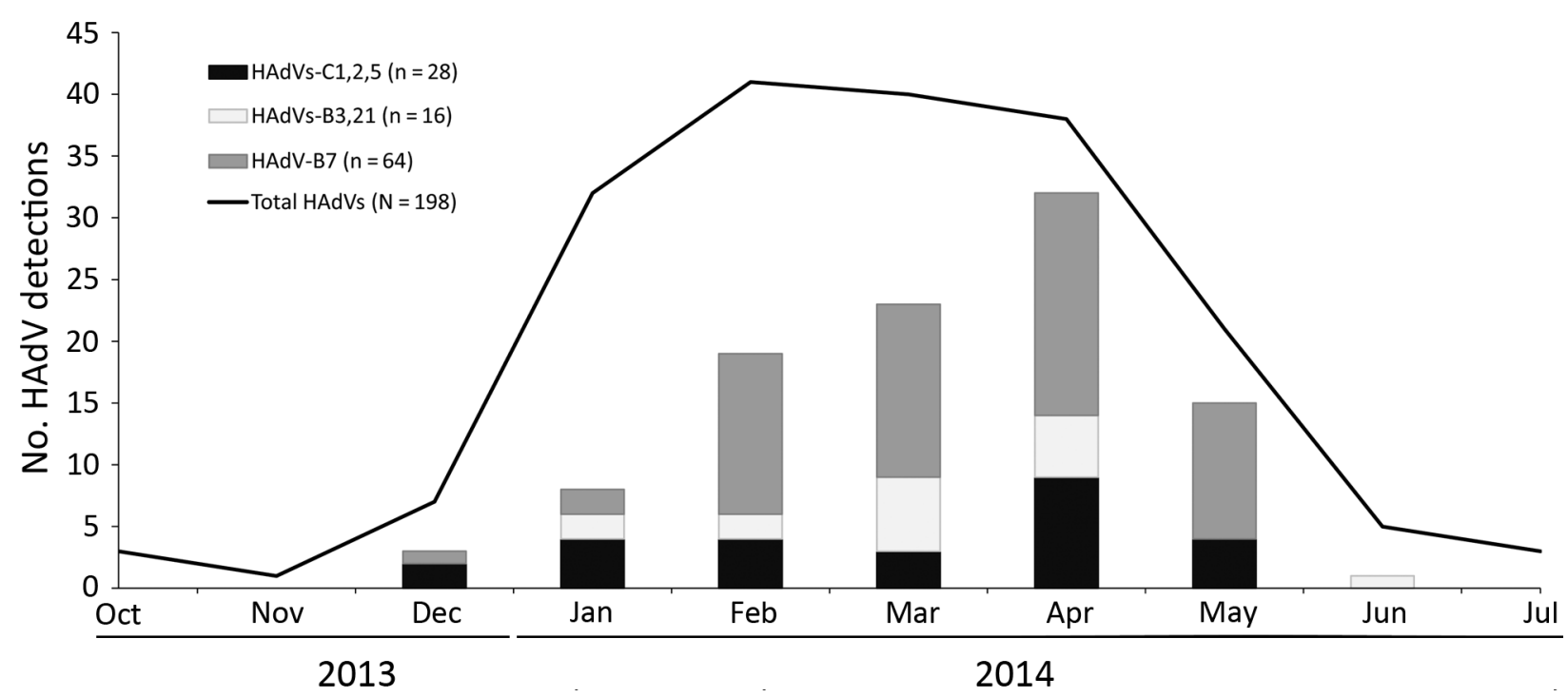

Figure 3. HAdV detections by type and by month of symptom onset, Oregon, USA, October 2013-July 2014. If month of symptom onset was not available, month of specimen collection was used. Total HAdVs include 109 HAdV-positive specimens that were typed (including 1 HAdV-E4 specimen) and 89 specimens that were not available for typing. HAdV, human adenovirus.

total of $136(69 \%)$ persons were hospitalized (Table 1), and $62(31 \%)$ were outpatients seen in the emergency department or in outpatient clinics. HAdV-B patients were more often hospitalized (HAdVs-B3 or -B21: 81\%; HAdV-B7: $84 \%)$ than HAdVs-C1, -C2, or -C5 (64\%) patients (Table 1). Among hospitalized case-patients, $32 \%$ were admitted to the ICU, and $18 \%$ required mechanical ventilation (Table 1); $46 \%$ of HAdV-B7 case-patients were admitted to the ICU (Table 1). Median length of hospital stay for HAdV-B7 case-patients was longer (6.5 days; interquartile range [IQR] 3-9.5 days) than for HAdVs-B3 or -B21 casepatients (5.0 days; IQR 3-5 days) and HAdVs-C1, -C2, or -C5 case-patients (2.5 days; IQR 2-4 days). However, when we compared HAdV-B7 with non-HAdV-B7 casepatients, only length of hospitalization remained statistically significant after adjustment for age; HAdV-B7 casepatients were significantly older (adults) and hospitalized longer than the median (4 days) than non-HAdV-B7 casepatients $(p<0.05)$ (Table 2$)$. Five $(2.5 \%)$ case-patients died; for 2, specimens were available for typing, and both were HAdV-B7.

\section{Discussion}

We describe a community outbreak of HAdV in Oregon where HAdV-B7 was identified as the predominant HAdV type. HAdV-B7 was more often detected in adults than children and was associated with more severe disease than other HAdV types. In persons $\leq 18$ years of age, $7 \mathrm{HAdV}$ types were detected, whereas in most persons $>18$ years of age, HAdVs-B3, -B7, or -B21 were detected. These findings are consistent with other studies showing that HAdVs-C1,
$-\mathrm{C} 2$, and -C5 are most frequently detected in young children $(17,25)$. Many studies have demonstrated severe illness associated with HAdV-B7 in children or in military recruits, but few have documented such a widespread community outbreak in which many adults experience severe respiratory illness $(5,18,26,27)$. However, a high percentage of adults with severe respiratory disease has been reported in other community outbreaks of related HAdV-B viruses $(6,13,28,29)$.

Compared with non-HAdV-B7 case-patients, HAdVB7 case-patients were more frequently admitted to the ICU and mechanically ventilated, were significantly older, and had significantly longer hospital stays. Our findings of severe infection with $\mathrm{HAdV}$-B7 requiring ICU admission and extended hospitalization is consistent with other reports linking HAdV-B7 with more severe acute respiratory disease (30-32). HAdV also was detected in 5 fatal cases, 2 of which were identified as HAdV-B7. Although the contribution of HAdV to a patients' death is unclear, similar reports of severe outcomes associated with HAdV infection have been documented $(18,26,30)$.

Further molecular analysis showed that genome sequences of 7 Oregon HAdV-B7 isolates were identical to each other and nearly identical to strains circulating in China in 2009 and 2011. Predicted restriction enzyme profiles have not previously identified the genome of this virus (HAdV-B7d) in the United States (A. Kajon, pers. comm.) until recently (33). HAdV-B7d was first isolated in China in 1980, where it became the predominant circulating genome type at least through 1990 (22) but then disappeared until reemerging 21 years later (15). Severe respiratory 
Table 1. Demographic and clinical characteristics for HAdV-positive case-patients, Oregon, October 2013-July 2014*

\begin{tabular}{|c|c|c|c|c|c|}
\hline Characteristic & Total & HAdVs-B3, -B21 & HAdV-B7 & HAdVs-C1, $-2,-5$ & Not available† \\
\hline Total & $198(100)$ & $16(8.1)$ & $64(32.3)$ & $28(14.1)$ & $89(45.0)$ \\
\hline \multicolumn{6}{|l|}{ Age group, y } \\
\hline Children, $\leq 18$ y & $118(59.6)$ & $8(50.0)$ & $28(43.8)$ & $27(96.4)$ & $54(60.7)$ \\
\hline$<2$ & $63(31.8)$ & $3(18.8)$ & $16(25.0)$ & $25(89.3)$ & $19(21.4)$ \\
\hline $2-5$ & $24(12.1)$ & $4(25.0)$ & $5(7.8)$ & $1(3.6)$ & $14(15.7)$ \\
\hline $6-10$ & $20(10.1)$ & 0 & $4(6.3)$ & $1(3.6)$ & $15(16.9)$ \\
\hline $11-18$ & $11(5.6)$ & $1(6.3)$ & $3(4.7)$ & 0 & $6(6.7)$ \\
\hline Adults, $>18$ y & $80(40.4)$ & $8(50.0)$ & 36 (56.3) & $1(3.6)$ & $35(39.3)$ \\
\hline $19-25$ & $16(8.1)$ & 0 & $6(9.4)$ & 0 & $10(11.2)$ \\
\hline $26-45$ & $19(9.6)$ & $2(12.5)$ & $10(15.6)$ & 0 & $7(7.9)$ \\
\hline $46-65$ & $35(17.7)$ & $6(37.5)$ & $18(28.1)$ & $1(3.6)$ & $10(11.2)$ \\
\hline$>65$ & $10(5.1)$ & 0 & $2(3.1)$ & 0 & $8(9.0)$ \\
\hline \multicolumn{6}{|l|}{ Common symptoms at presentation } \\
\hline Fever & $149(75.3)$ & $12(75.0)$ & $50(78.2)$ & $18(64.3)$ & $69(77.5)$ \\
\hline Cough & $121(61.1)$ & $10(62.5)$ & $45(70.3)$ & $18(64.3)$ & $48(53.9)$ \\
\hline Shortness of breath & $52(26.3)$ & $7(43.8)$ & $23(35.9)$ & $7(25)$ & $15(16.9)$ \\
\hline Nausea or vomiting & $47(23.7)$ & $4(25.0)$ & $14(21.9)$ & $9(32.1)$ & $20(22.5)$ \\
\hline Rhinorrhea & $43(21.7)$ & $5(31.3)$ & $6(9.4)$ & $10(35.7)$ & $22(24.7)$ \\
\hline Fatigue & $27(13.6)$ & $2(12.5)$ & $15(23.4)$ & $3(10.7)$ & $7(7.9)$ \\
\hline Diarrhea & $22(11.1)$ & $3(18.8)$ & $9(14.1)$ & $2(7.1)$ & $8(9.0)$ \\
\hline Sore throat & $20(10.1)$ & $2(12.5)$ & $3(4.7)$ & 0 & $15(16.9)$ \\
\hline Myalgia & $20(10.1)$ & $1(6.3)$ & $6(9.4)$ & 0 & $13(14.6)$ \\
\hline Wheezing & $13(6.6)$ & $3(18.8)$ & $3(4.7)$ & $4(14.3)$ & $3(3.4)$ \\
\hline \multicolumn{6}{|l|}{ Illness severity } \\
\hline Hospitalized & $136(68.7)$ & $13(81.3)$ & $54(84.4)$ & $18(64.3)$ & $51(57.3)$ \\
\hline Admitted to ICU $\ddagger$ & $43(31.6)$ & $4(30.8)$ & $25(46.3)$ & $4(22.2)$ & $10(19.6)$ \\
\hline Required mechanical ventilation $\ddagger$ & $25(18.4)$ & $3(23.1)$ & $13(24.1)$ & $1(3.6)$ & $8(15.7)$ \\
\hline Died & $5(2.5)$ & 0 & $2(3.1)$ & 0 & $3(3.4)$ \\
\hline Age, y, mean/median (IQR)§ & $20.6 / 8.0(1.9-40)$ & $26.8 / 24.0(3-50.5)$ & $26.8 / 20.0(2.5-50.5)$ & $3.5 / 1.2(0.7-1.6)$ & $20.6 / 9.0(3-29)$ \\
\hline $\begin{array}{l}\text { Days from symptom onset to } \\
\text { hospital admission, mean/median } \\
\text { (IQR) } \ddagger \S\end{array}$ & $4.2 / 3.0(2-5)$ & $3.6 / 3.0(2-4)$ & $4.6 / 3.0(2-6)$ & $3.9 / 3.0(1-4)$ & $4.2 / 4.0(2-5)$ \\
\hline $\begin{array}{l}\text { Days hospitalized, mean/median } \\
(\mathrm{IQR}) \ddagger \S\end{array}$ & $6.5 / 4.0(2-8)$ & $5.0 / 5.0(3-5)$ & $8.4 / 6.5(3-9.5)$ & $3.4 / 2.5(2-4)$ & $6.3 / 4.0(2-6)$ \\
\hline \multicolumn{6}{|c|}{$\begin{array}{l}\text { "Values are no. (\%) except as indicated. Total HAdV also includes HAdV-E4 (1 case), which was in a nonhospitalized child (10-18 y). HAdV, human } \\
\text { adenovirus; ICU, intensive care unit; IQR, interquartile range. } \\
\text { †Specimens from these HAdV-positive patients were not available for typing. } \\
\text { †Among hospitalized HAdV patients. } \\
\text { §IQR }=25 \% \text { and } 75 \% \text { quartiles. }\end{array}$} \\
\hline
\end{tabular}

disease and higher case-fatality rates have been associated with HAdV-B7, especially HAdV-B7d. Enhanced virus fitness, low herd immunity to HAdV-B7, or both might have contributed to the large community outbreak in Oregon.

The US military used an effective live oral vaccine for HAdV-E4 and HAdV-B7 from 1971 until 1999, and the vaccine was reintroduced in $2011(34,35)$. During 1999-2011, when the vaccine was unavailable, vaccinepreventable HAdV infections increased substantially in US military personnel (36). Subsequent reintroduction of the vaccine in October 2011 resulted in a significant decrease in these HAdV infections, indicating HAdV vaccination is an effective prevention measure for HAdV infection in this setting (35). However, currently no HAdV vaccine is available for use in the general public.

Our study has several limitations. The HAdV cases described in this case series do not represent a populationbased sample and might not represent all HAdV cases in Oregon during this period. Persons with milder illness were not sought out at participating facilities because case finding was primarily conducted in hospitals and because the
Health Alert Network notice requested providers to consider HAdV in patients with severe pneumonia. Because of the timing of the investigation, almost half of the HAdV-positive specimens were not available for typing (many were cases diagnosed earlier in the study period), and $80 \%$ of these specimens came from 1 of the 3 major hospital systems that participated in the investigation. The molecular methods used for typing HAdVs in this study targeted the hexon hypervariable regions that have been shown to correlate closely with virus serotype. These methods do not provide genomic detail and might miss recombination events in other regions of the virus genome. Moreover, full-genome sequencing was performed on only 7 HAdV-B7 isolates, and although they were chosen from different time points in the outbreak, we cannot conclude that all HAdV-B7 detections were HAdV-B7d. Finally, detection of HAdV in a respiratory specimen, especially HAdV-C, does not necessarily imply causation because asymptomatic HAdV shedding can occur. HAdV-C detections in infants and young children with mild respiratory infections and the virus might persist in the adenoids and tonsils and be shed for prolonged periods 


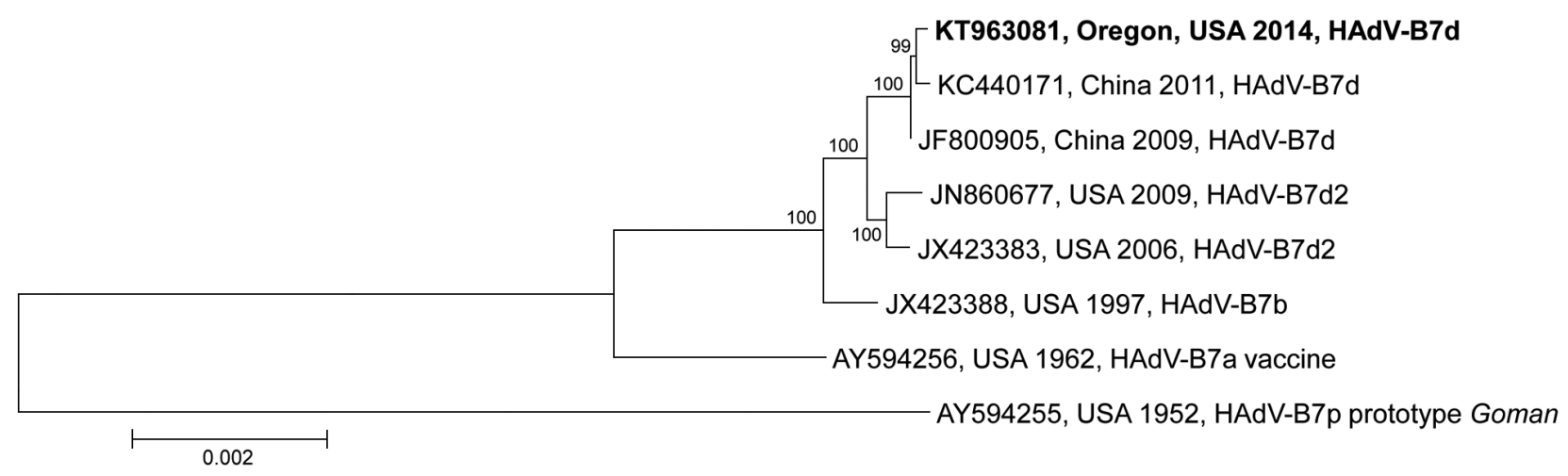

Figure 4. Phylogenetic analysis of human adenovirus 7 genome type d (HAdV-B7d), Oregon, USA, 2014. Genomic sequences were aligned by using ClustalW implemented in BioEdit version 7.2.5 and the neighbor-joining phylogenetic tree constructed by using MEGA7 software $(23,24)$. Numbers at selected nodes indicate level of support using 1,000 bootstrap replicates. Sequences are identifoed by GenBank accession number, geographic location, year of sample collection, and virus genome type identified. Boldface indicates 7 identical genomic sequences identified during this study; sequences are from HAdV-B7 isolates derived from 7 different cases spanning the outbreak period. Scale bar indicates estimated number of nucleotide substitutions per site.

after symptoms resolve (37-39). The clinical significance of HAdV latency in tonsil and adenoid tissue is unclear.

HAdV type surveillance is an important tool for monitoring changes in predominant types and genome types. Shifts in HAdV types might be associated with more severe disease not only in vulnerable populations, such as children, elderly persons, and immunocompromised persons, but might also cause community outbreaks of severe respiratory disease in adults, as occurred in Oregon. Healthcare providers should consider HAdV in their differential diagnosis for patients with pneumonia and acute respiratory infection. Testing for HAdV using respiratory panel PCR assays and HAdV typing has been increasing nationwide. In response, $\mathrm{CDC}$ recently launched a voluntary and passive surveillance system to collect HAdV typing data from laboratories called the National Adenovirus Type Reporting System. The main objectives of this system are to better define circulation patterns of HAdV types and better monitor HAdV outbreaks in the United States.
HAdV-B7 might be reemerging in the United States and might be associated with increased numbers of severe respiratory infections. Tracking the emergence of HAdV types in the United States will lead to early identification of new types and potential variants of known types. Our results demonstrate how HAdV surveillance might help explain clusters and sporadic cases of severe illness possibly related to changes in HAdV species.

\section{Acknowledgments}

We gratefully acknowledge the invaluable contributions of the staff at the hospitals submitting data and specimens for this research, as well as the counties and laboratory staff assisting OPHD: Margaret Oethinger, Mary Campbell, Margaret Ford, Claudia Atherton, Robert Liao, Jana Brott, Kelly Reed, Patrick Luedtke, Laura Reynolds, Maayan Simckes, Misty Lang, Malini DeSilva, and Marjorie Yungclas.

Ms. Kendall Scott is the influenza epidemiologist for OPHD. Her research interests include influenza and other respiratory viruses.

\begin{tabular}{|c|c|c|c|}
\hline Characteristic & HAdV-B7, no. (\%) & Non-HAdV-B7, no. $(\%) \dagger$ & $\mathrm{p}$ value \\
\hline Total & 64 & 45 & \\
\hline \multicolumn{4}{|l|}{ Age group, y } \\
\hline Children, $\leq 18$ & $28(43.7)$ & $36(80.0)$ & $<0.001$ \\
\hline Adults, $>18$ & $36(56.3)$ & $9(20.0)$ & \\
\hline \multicolumn{4}{|l|}{ Illness severity } \\
\hline Hospitalized & $54(84.4)$ & $31(68.9)$ & $<0.05 \ddagger$ \\
\hline Admitted to ICU§ & $25(46.3)$ & $8(25.8)$ & 0.06 \\
\hline Required mechanical ventilation§ & $13(24.1)$ & $4(12.9)$ & 0.26 \\
\hline \multicolumn{4}{|l|}{ Days hospitalized } \\
\hline <Median, 4 & $14(35.0)$ & $19(70.4)$ & $<0.05$ \\
\hline$>$ Median, 4 & $26(65.0)$ & $8(29.6)$ & \\
\hline
\end{tabular}

*HAdV, human adenovirus; ICU, intensive care unit

tIncludes only the 109 specimens that were typed. Non-HAdV-B7: HAdVs-B3, -B21, HAdVs-C1, -C2, -C5, and HAdV-E4.

$\ddagger$ Not significant after adjustment for age.

§Among hospitalized case-patients.

TA total of 40 HAdV-B7 case-patients and 27 non-HAdV-B7 case-patients had both hospital admission and discharge dates. 


\section{References}

1. Ison MG. Adenovirus infections in transplant recipients. Clin Infect Dis. 2006;43:331-9. http://dx.doi.org/10.1086/505498

2. Lee J, Choi EH, Lee HJ. Clinical severity of respiratory adenoviral infection by serotypes in Korean children over 17 consecutive years (1991-2007). J Clin Virol. 2010;49:115-20. http://dx.doi.org/ 10.1016/j.jcv.2010.07.007

3. Kandel R, Srinivasan A, D’Agata EMC, Lu X, Erdman D, Jhung M. Outbreak of adenovirus type 4 infection in a long-term care facility for the elderly. Infect Control Hosp Epidemiol. 2010; 31:755-7. http://dx.doi.org/10.1086/653612

4. Moura PO, Roberto AF, Hein N, Baldacci E, Vieira SE, Ejzenberg B, et al. Molecular epidemiology of human adenovirus isolated from children hospitalized with acute respiratory infection in São Paulo, Brazil. J Med Virol. 2007;79:174-81. http://dx.doi.org/10.1002/jmv.20778

5. Gerber SI, Erdman DD, Pur SL, Diaz PS, Segreti J, Kajon AE, et al. Outbreak of adenovirus genome type $7 \mathrm{~d} 2$ infection in a pediatric chronic-care facility and tertiary-care hospital. Clin Infect Dis. 2001;32:694-700. http://dx.doi.org/10.1086/319210

6. Lessa FC, Gould PL, Pascoe N, Erdman DD, Lu X, Bunning ML, et al. Health care transmission of a newly emergent adenovirus serotype in health care personnel at a military hospital in Texas, 2007. J Infect Dis. 2009;200:1759-65. http://dx.doi.org/ $10.1086 / 647987$

7. Lewis PF, Schmidt MA, Lu X, Erdman DD, Campbell M, Thomas A, et al. A community-based outbreak of severe respiratory illness caused by human adenovirus serotype 14 . J Infect Dis. 2009;199:1427-34. http://dx.doi.org/10.1086/598521

8. Calder JA, Erdman DD, Ackelsberg J, Cato SW, Deutsch VJ, Lechich AJ, et al. Adenovirus type 7 genomic-type variant, New York City, 1999. Emerg Infect Dis. 2004;10:149-52. http://dx.doi.org/10.3201/eid1001.020605

9. Heim A, Ebnet C, Harste G, Pring-Åkerblom P. Rapid and quantitative detection of human adenovirus DNA by real-time PCR. J Med Virol. 2003;70:228-39. http://dx.doi.org/10.1002/jmv.10382

10. Lion T. Adenovirus infections in immunocompetent and immunocompromised patients. Clin Microbiol Rev. 2014;27:44162. http://dx.doi.org/10.1128/CMR.00116-13

11. Metzgar D, Osuna M, Kajon AE, Hawksworth AW, Irvine M, Russell KL. Abrupt emergence of diverse species B adenoviruses at US military recruit training centers. J Infect Dis. 2007;196:146573. http://dx.doi.org/10.1086/522970

12. Lebeck MG, McCarthy TA, Capuano AW, Schnurr DP, Landry ML, Setterquist SF, et al. Emergent US adenovirus 3 strains associated with an epidemic and serious disease. J Clin Virol. 2009;46:331-6. http://dx.doi.org/10.1016/j.jcv.2009.09.023

13. Abbas KZ, Lombos E, Duvvuri VR, Olsha R, Higgins RR, Gubbay JB. Temporal changes in respiratory adenovirus serotypes circulating in the greater Toronto area, Ontario, during December 2008 to April 2010. Virol J. 2013;10:15. http://dx.doi.org/10.1186/ $1743-422 \mathrm{X}-10-15$

14. Ng OT, Thoon KC, Chua HY, Tan NW, Chong CY, Tee NW, et al. Severe pediatric adenovirus 7 disease in Singapore linked to recent outbreaks across Asia. Emerg Infect Dis. 2015;21:1192-6. http://dx.doi.org/10.3201/eid2107.141443

15. Zhao S, Wan C, Ke C, Seto J, Dehghan S, Zou L, et al. Re-emergent human adenovirus genome type $7 \mathrm{~d}$ caused an acute respiratory disease outbreak in Southern China after a twenty-one year absence. Sci Rep. 2014;4:7365. http://dx.doi.org/10.1038/ srep07365

16. Tsou TP, Tan BF, Chang HY, Chen WC, Huang YP, Lai CY, et al. Community outbreak of adenovirus, Taiwan, 2011. Emerg Infect Dis. 2012;18:1825-32. http://dx.doi.org/10.3201/eid1811.120629

17. Gray GC, McCarthy T, Lebeck MG, Schnurr DP, Russell KL, Kajon AE, et al. Genotype prevalence and risk factors for severe clinical adenovirus infection, United States 2004-2006. Clin Infect Dis. 2007;45:1120-31. http://dx.doi.org/10.1086/522188

18. Selvaraju SB, Kovac M, Dickson LM, Kajon AE, Selvarangan R. Molecular epidemiology and clinical presentation of human adenovirus infections in Kansas City children. J Clin Virol. 2011;51:126-31. http://dx.doi.org/10.1016/j.jcv.2011.02.014

19. Lu X, Trujillo-Lopez E, Lott L, Erdman DD. Quantitative real-time PCR assay panel for detection and type-specific identification of epidemic respiratory human adenoviruses. J Clin Microbiol. 2013;51:1089-93. http://dx.doi.org/10.1128/ JCM.03297-12

20. Lu X, Erdman DD. Molecular typing of human adenoviruses by PCR and sequencing of a partial region of the hexon gene. Arch Virol. 2006;151:1587-602. http://dx.doi.org/10.1007/s00705-005-0722-7

21. Vincze T, Posfai J, Roberts RJ. NEBcutter: a program to cleave DNA with restriction enzymes. Nucleic Acids Res. 2003;31:368891. http://dx.doi.org/10.1093/nar/gkg526

22. Li QG, Zheng QJ, Liu YH, Wadell G. Molecular epidemiology of adenovirus types 3 and 7 isolated from children with pneumonia in Beijing. J Med Virol. 1996;49:170-7 http://dx.doi.org/10.1002/ (SICI)1096-9071(199607)49:3<170::AID-JMV3>3.0.CO;2-1.

23. Hall TA. BioEdit: a user-friendly biological sequence alignment editor and analysis program for Windows 95/98/NT. Nucleic Acids Symp Ser. 1999;41:95-8 [cited 2016 Mar 18]. http://www.researchgate.net/publication/200038090_BioEdit_A_ user-friendly biological sequence alignment program for Windows_9598NT

24. Tamura K, Stecher G, Peterson D, Filipski A, Kumar S. MEGA6: Molecular Evolutionary Genetics Analysis version 6.0. Mol Biol Evol. 2013;30:2725-9. http://dx.doi.org/10.1093/molbev/mst197

25. Hong JY, Lee HJ, Piedra PA, Choi EH, Park KH, Koh YY, et al. Lower respiratory tract infections due to adenovirus in hospitalized Korean children: epidemiology, clinical features, and prognosis. Clin Infect Dis. 2001;32:1423-9. http://dx.doi.org/ $10.1086 / 320146$

26. Ryan MAK, Gray GC, Smith B, McKeehan JA, Hawksworth AW, Malasig MD. Large epidemic of respiratory illness due to adenovirus types 7 and 3 in healthy young adults. Clin Infect Dis. 2002;34:577-82. http://dx.doi.org/10.1086/338471

27. Yu P, Ma C, Nawaz M, Han L, Zhang J, Du Q, et al. Outbreak of acute respiratory disease caused by human adenovirus type 7 in a military training camp in Shaanxi, China. Microbiol Immunol. 2013;57:553-60. http://dx.doi.org/10.1111/1348-0421.12074

28. Centers for Disease Control and Prevention. Acute respiratory disease associated with adenovirus serotype 14-four states, 2006-2007. MMWR Morb Mortal Wkly Rep. 2007;56:1181-4.

29. Gu L, Liu Z, Li X, Qu J, Guan W, Liu Y, et al. Severe communityacquired pneumonia caused by adenovirus type 11 in immunocompetent adults in Beijing. J Clin Virol. 2012;54:295301. http://dx.doi.org/10.1016/j.jcv.2012.04.018

30. Carballal G, Videla C, Misirlian A, Requeijo PV, Aguilar MC. Adenovirus type 7 associated with severe and fatal acute lower respiratory infections in Argentine children. BMC Pediatr. 2002; 2:6. http://dx.doi.org/10.1186/1471-2431-2-6

31. Cui X, Wen L, Wu Z, Liu N, Yang C, Liu W, et al. Human adenovirus type 7 infection associated with severe and fatal acute lower respiratory illness and nosocomial transmission. J Clin Microbiol. 2015;53:746-9. http://dx.doi.org/10.1128/ JCM.02517-14

32. Mitchell LS, Taylor B, Reimels W, Barrett FF, Devincenzo JP. Adenovirus 7a: a community-acquired outbreak in a children's hospital. Pediatr Infect Dis J. 2000;19:996-1000. http://dx.doi.org/10.1097/00006454-200010000-00011

33. Kajon AE, Ison MG. Severe infections with adenovirus $7 \mathrm{~d}$ in 2 adults in family, Illinois, USA, 2014. Emerg Infect Dis. 2016;22:730-3. http://dx.doi.org/10.3201/eid2204.151403 
34. Top FH Jr, Dudding BA, Russell PK, Buescher EL. Control of respiratory disease in recruits with types 4 and 7 adenovirus vaccines. Am J Epidemiol. 1971;94:142-6.

35. Radin JM, Hawksworth AW, Blair PJ, Faix DJ, Raman R, Russell KL, et al. Dramatic decline of respiratory illness among US military recruits after the renewed use of adenovirus vaccines. Clin Infect Dis. 2014;59:962-8. http://dx.doi.org/10.1093/cid/ciu507

36. Gray GC. Adenovirus vaccines. In: Plotkin SA, Orenstein WA, Offit PA, editors. Vaccines. 6th ed. London: WB Saunders; 2013. p. 113-126.

37. Garnett CT, Erdman D, Xu W, Gooding LR. Prevalence and quantitation of species $\mathrm{C}$ adenovirus DNA in human mucosal lymphocytes. J Virol. 2002;76:10608-16. http://dx.doi.org/10.1128/ JVI.76.21.10608-10616.2002
38. Alkhalaf MA, Guiver M, Cooper RJ. Prevalence and quantitation of adenovirus DNA from human tonsil and adenoid tissues. J Med Virol. 2013;85:1947-54. http://dx.doi.org/10.1002/ jmv. 23678

39. Neumann R, Genersch E, Eggers HJ. Detection of adenovirus nucleic acid sequences in human tonsils in the absence of infectious virus. Virus Res. 1987;7:93-7. http://dx.doi.org/10.1016/ 0168-1702(87)90060-8

Address for correspondence: Magdalena Kendall Scott, Oregon Public Health Division, 800 NE Oregon St, Portland, OR 97232, USA; email: magdalena.k.scott@state.or.us

\section{November 2015: Ebola Including:}

- Ebola in West Africa-CDC's Role in Epidemic Detection, Control, and Prevention

- Use of Internet Search Queries to Enhance Surveillance of Foodborne Illness

- Achievements and Challenges of Tuberculosis Control in South Korea

- Ebola Virus Outbreak Investigation, Sierra Leone, September 28-November 11, 2014

- Neurologic Disorders in Immunocompetent Patients with Autochthonous Acute Hepatitis E

- Mycotic Infections Acquired outside Areas of Endemicity, United States

- Uncommon Candida Species Fungemia among Cancer Patients, Houston, Texas, USA

- Maternal Effects of Respiratory Syncytial Virus Infection during Pregnancy

- Serotype PDF Linked and Drug Resistance in Invasive Pneumococcal Diseases in Adults after Vaccinations in Children, Japan, 2010-2013

- Carbapenem-Resistant Enterobacteriaceae in Children, United States, 1999-2012

- Role of Maternal Antibodies in Infants with Severe Diseases Related to Human Parechovirus

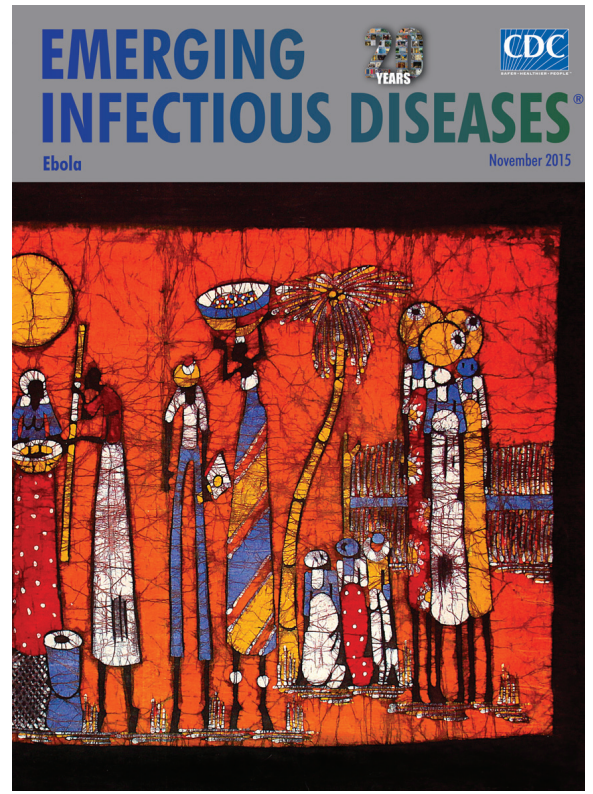

- Type 3USA300 Methicillin-Resistant Staphylococcus aureus, United States, 2000-2013

- Molecular Epidemiology of Hospital Outbreak of Middle East Respiratory Syndrome, Riyadh, Saudi Arabia, 2014

- Climatic Influences on Cryptoccoccus gattii Populations, Vancouver Island, Canada, 2002-2004

- Coccidioidomycosis among Workers Constructing Solar Power Farms, California, USA, 2014

- Shigella Infections in Household Contacts of Pediatric Shigellosis Patients in Rural

- Contact Tracing Activities during the Ebola Virus Disease Epidemic in Kindia and Faranah, Guinea, 2014

- Association of Higher MERS-CoV Virus Load with Severe Disease and Death, Saudi Arabia, 2014

http://wwwnc.cdc.gov/eid/articles/issue/ $21 / 11 /$ table-of-contents 